\title{
Job burnout and turnover intention among nurses in China: the mediating effects of positive emotion $^{\dagger}$
}

\author{
Li-Feng Yang, Jing-Ying Liu, Yan-Hui Liu*
}

School of Nursing, Tianjin University of Traditional Chinese Medicine, Tianjin 300193, China

Received: 18 September 2017; Accepted: 14 October 2017; Published: 20 March 2018

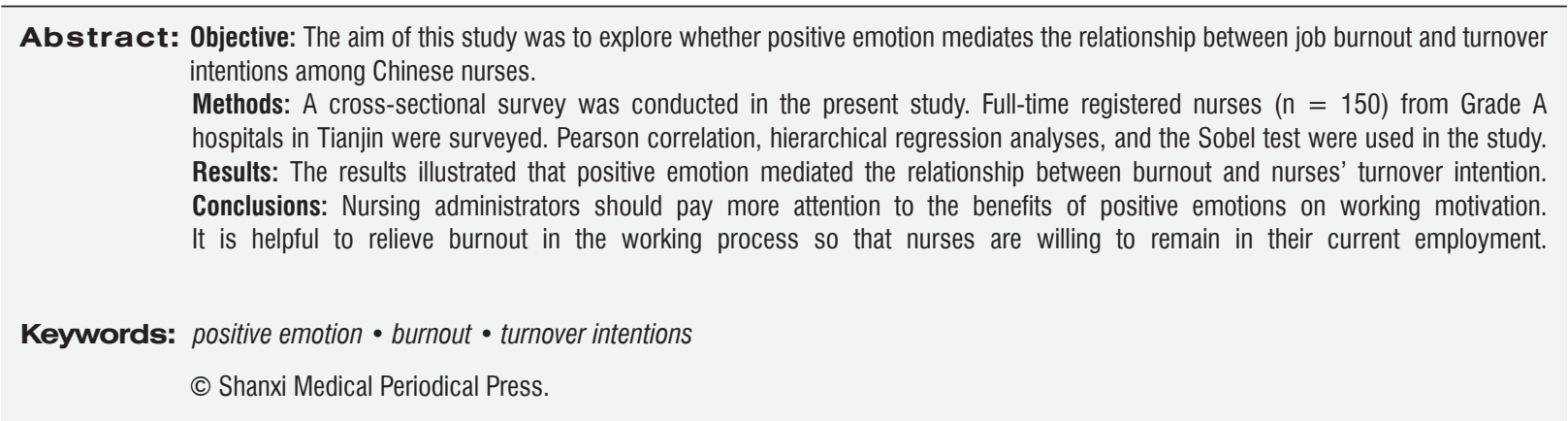

\section{Introduction}

It is well known that the health-care system is confronted with a shortage of nurses in the workforce and that it is facing rapidly increasing needs for patient care in an aging population..$^{1,2}$ This situation has made the nursing profession more stressful and susceptible to turnover. ${ }^{3}$ Studies have shown that the average turnover rate for nurses in Canada is $19.9 \% .{ }^{4}$ Nurses' intention to leave their profession varied from $5 \%$ to $17 \%$ in European countries. ${ }^{5}$ Slightly more than half $(50.2 \%)$ of the nurses in Ethiopia reported the same intention. ${ }^{6}$ China is a populous country, but it also has an aging population; the circumstances may be more serious in China. Turnover intention is the main predictor of turnover action, ${ }^{7}$ which affects not only nurses' mental health but also their work efficiency

\footnotetext{
${ }^{\dagger}$ This project was supported by the National Natural Science Foundation (No. 71704132).
}

*E-mail:yh_liu888@163.com and service quality. In recent years, there have been studies about turnover intention in many countries. Most studies focus on risk factors and negative interventions associated with turnover intentions. Hence, the research on individuals' active initiatives remains to be enriched.

Burnout is defined as a response to ongoing stress or psychological strain. ${ }^{8}$ Employee burnout can trigger turnover challenges for leaders and institutions. Previous literature has reported that burnout positively affects turnover intentions. "Turnover intentions" refers to a psychological tendency that could lead to turnover behaviors when employees are not satisfied in their work. ${ }^{10}$

With the rise of positive psychology, researchers have begun to explore job burnout and turnover intention from a new perspective, highlighting the development of individual potential and the exploration of mental resources. ${ }^{11}$ "Positive emotions" is a category of positive psychology. It refers to an individual's subjective experi- 
ence and indicates a temporary or persistently positive emotional state. It can increase psychological flexibility and help a person maintain good mental health. Previous literature has reported that positive emotions are significantly, negative correlated with burnout, ${ }^{12}$ possibly reducing burnout and serving as the controlling factor in burnout. Thus, we suggest that positive emotions mediate the relationship between burnout and turnover intentions.

Recently, there have been many studies written from the perspective of positive psychology, which focus on ways to decrease turnover intentions. ${ }^{13,14}$ The purpose of our study was to investigate the relationships among burnout, positive emotions, and turnover intentions in nurses in China. We wanted to know the influence of burnout and positive emotions on nurses' turnover intentions.

\section{Methods}

\subsection{Design and sample}

A cross-sectional design with self-reported questionnaires was utilized in this survey. A sample of 150 registered nurses from Grade $A$ hospitals in Tianjin was surveyed. Electronic questionnaires and paper questionnaires were posted to approximately 200 nurses in August 2015. Because some nurses were busy with work and family, some questionnaires were not handed back. In all, 160 questionnaires were completed, a reclamation rate of $80 \%$. Ten of the completed questionnaires were excluded for missing data. Overall, the sample size included in this study was 150; the valid reclamation rate was $75 \%$. Females accounted for $98 \%$ of all participants; $18 \%$ of participants were $>25$ years of age, and $43.3 \%$ held a bachelor's degree.

\subsection{Instruments}

The research tools used in this study include four parts: (a) demographic information; (b) Turnover Intentions Scale; (c) Burnout Scale; and (d) Positive Affect and Negative Affect Scale.

\subsubsection{Nurses' demographic information}

Nurses' demographic data included personal details such as age, gender, marital status, and education level. These demographic data are potential variables influencing burnout, turnover intentions, and positive emotion.

\subsubsection{Burnout}

We measured nurses' burnout with the scale of the Chinese version of the Maslach Burnout Inventory - General Survey (MBI-GS), ${ }^{15}$ which consisted of 15 items and three dimensions, including emotional exhaustion, cynicism, and reduced professional efficacy. The Chinese version of MBI-GS has been verified as a high-reliability tool. ${ }^{16}$ In this study, the total Cronbach's a was 0.86 , and the Cronbach's $\alpha$ of the dimensions ranged from 0.82 to 0.88. Respondents answered using a seven-point Likert scale, ranging from zero ("never") to six ("every day"). Higher scores show a more serious level of burnout.

\subsubsection{Turnover intentions}

To evaluate the turnover intentions of nurses, the version of the three-items scale developed by Luthans and Jensen ${ }^{17}$ was used. Items included the following statements: "If I can choose now, I want to change to other work outside nursing; "As for me, my work is the best at present"; "Choosing to work here now seems like the wrong choice." Respondents were asked to rate each item by selecting one of six options, ranging from one (strongly disagree) to six (strongly agree). The scores were recorded, with higher scores indicating higher levels of intention to leave the hospital. The Cronbach's aof the total scale was 0.87 .

\subsubsection{Positive emotion}

Positive emotion was measured using the Chinese version of the Positive Affect and Negative Affect Scale (PANAS). ${ }^{18}$ We used only Positive Affect, which includes nine items. Respondents rated each item on a five-point Likert scale, ranging from one (not very strong) to five (very strong). In this study, the Cronbach's aof the scale was 0.85 . Higher scores indicated greater job enthusiasm.

\subsection{Ethical considerations}

This study has obtained the consent of the medical ethics committees of the participating hospitals and the authors' university. The participants were informed that involvement was absolutely voluntary and that they could drop out of the survey at will at any time with no adverse ramifications.

\subsection{Statistical analysis}

All analyses were performed using the SPSS 17.0 program, and all statistical tests were two-sided $(\alpha=0.05)$. 
Descriptive statistics were used to summarize sample characteristics. Pearson correlation analysis was performed for testing the relationships among all variables. Hierarchical regression was used for testing the hypotheses of the mediating effects of positive emotion on burnout and turnover intention. The Sobel test was further used to prove the significance of the mediating effects of positive emotion.

\section{Results}

\subsection{The demographic characteristics of nurses}

The demographic characteristics of the participants are shown in Table 1. The respondents included 150 nurses. Of these, 89 nurses were unmarried (59.3\%). Females accounted for $98 \%$ of the participants, $18 \%$ were $>25$ years of age, and $43.3 \%$ held a Bachelor's degree.

\begin{tabular}{lcr}
\hline \multicolumn{1}{c}{ Variables } & Number of participants & $\%$ \\
\hline \hline Gender & 147 & \\
Female & 3 & 98.0 \\
Male & & 2.0 \\
Marital status & 61 & \\
Married & 89 & 40.7 \\
Single & & 59.3 \\
Age group (in years) & 123 & \\
20-25 & 23 & 82.0 \\
26-30 & 4 & 15.3 \\
31-35 & & 2.7 \\
Education & 9 & \\
Technical secondary & & 6.0 \\
school & 76 & 50.7 \\
Junior college & 65 & 43.3 \\
Bachelor & & \\
\hline
\end{tabular}

Table 1. Demographic characteristics of the participants

\subsection{The mean values, standard deviations, and correlation analysis among burnout, positive emotions, and turnover intentions}

Pearson's correlation test was performed to examine the relationships among burnout, positive emotions, and turnover intentions of nurses. The results in terms of the mean values, standard deviations, and all the variables' Pearson correlations are illustrated in Table 2. These variables, including burnout, positive emotions, and turnover intentions, were statistically significant effects and showed correlated relationships.

\subsection{Testing the hypothesized mediating effects of positive emotions}

To verify the mediating effects of positive emotions between burnout and nurses' turnover intentions, a multistep process was applied by Wen et al. ${ }^{19}$ Under the influence of controlling demographic variables, we examined the possible mediating effect of positive emotions on nurses' burnout and turnover intentions. The first step was to investigate the predictive effect of job burnout on turnover intentions. The second step was to investigate the predictive effect of job burnout on positive emotions. The third step was to investigate the predictive effect of job burnout and positive emotion on turnover intentions. Table 3 shows that job burnout can effectively predict turnover intentions and positive emotions after controlling for the influence of demographic data. Further analysis showed that the predictive effect of job burnout on turnover intentions is still significant, but the predictive power is reduced; the prediction effect of positive emotion on turnover intentions is significant after controlling for the influence of demographic variables and positive emotion. Positive emotion had a partial mediation effect between nurses' job burnout and turnover intentions, and the mediation effect accounted for $35 \%$ of the total effect.

\begin{tabular}{|c|c|c|c|c|c|c|c|c|}
\hline & M & SD & 1 & 2 & 3 & 4 & 5 & 6 \\
\hline 1. Burnout & 67.17 & 6.74 & 1 & - & - & - & - & - \\
\hline 2. Exhaustion & 19.21 & 1.62 & 0.045 & 1 & - & - & - & - \\
\hline 3. Cynicism & 15.71 & 1.70 & 0.030 & 0.124 & 1 & - & - & - \\
\hline 4. Professional efficacy & 24.93 & 1.89 & -0.125 & -0.016 & $-0.163^{\star}$ & 1 & - & - \\
\hline 5. Positive emotion & 15.35 & 2.40 & $-0.826^{\star \star}$ & -0.013 & -0.010 & 0.084 & 1 & - \\
\hline 6. Turnover intention & 16.29 & 0.62 & $0.926 * \star$ & 0.001 & 0.047 & -0.080 & $-0.893^{\star *}$ & 1 \\
\hline
\end{tabular}

Table 2. Pearson's correlation test of relationships among burnout, positive emotions, and turnover intentions of nurses Notes: ${ }^{*} P<0.05 ;{ }^{* *} P<0.01$. $\mathrm{M}=$ mean value; $\mathrm{SD}=$ standard deviation. 


\begin{tabular}{|c|c|c|c|c|c|c|c|c|c|}
\hline \multirow{2}{*}{ Variables } & \multicolumn{3}{|c|}{ Step 1: turnover intentions } & \multicolumn{3}{|c|}{ Step 2: positive emotion } & \multicolumn{3}{|c|}{ Step 3: turnover intentions } \\
\hline & $B$-value & SE & $\beta$-value & $B$-value & $S E$ & $\beta$-value & $B$-value & SE & $\beta$-value \\
\hline Gender & -0.121 & 0.135 & -0.027 & 0.529 & 0.812 & 0.031 & -0.067 & 0.108 & -0.015 \\
\hline Age & 0.030 & 0.010 & $0.108^{* \star}$ & -0.049 & 0.058 & -0.045 & 0.025 & 0.008 & $0.091^{* *}$ \\
\hline Marital status & -0.043 & 0.043 & -0.035 & 0.210 & 0.259 & 0.043 & -0.022 & 0.035 & -0.018 \\
\hline Education & 0.053 & 0.033 & 0.051 & -0.180 & 0.197 & -0.045 & 0.035 & 0.026 & 0.034 \\
\hline Burnout & 0.085 & 0.003 & $0.926^{\star *}$ & -0.295 & 0.017 & $-0.829^{\star \star}$ & 0.055 & 0.004 & $0.602^{* *}$ \\
\hline $\begin{array}{l}\text { Positive } \\
\text { emotion }\end{array}$ & - & - & - & - & - & - & -0.100 & 0.011 & $-0.391^{* *}$ \\
\hline$R^{2}$-value & & 0.868 & & & 0.688 & & & 0.916 & \\
\hline$F$-value & & $189.948^{\star \star}$ & & & $63.384^{\star *}$ & & & $260.429 * \star$ & \\
\hline
\end{tabular}

Table 3. Mediating effects of positive emotion on burnout and nurses' turnover intentions Notes: ${ }^{*} P<0.01 ; \mathrm{SE}=$ standard error; $\mathrm{B}$-value $=$ unstandardized $\mathrm{B}$.

Furthermore, we examined the significance of the mediating effects of positive emotion through a Sobel test. ${ }^{20}$ The results fully supported the hypothesis that positive emotion mediates burnout and nurses' turnover intentions $(z=2.69 ; P=0.001)$.

\section{Discussion}

In our study, the job burnout of Chinese nurses was more serious, and the level of turnover intentions was higher. This suggested that Chinese nurses' turnover intentions should not be ignored, and that it is imperative to find an effective intervention strategy. Our results also showed that burnout was positively associated with nurses' turnover intentions, results consistent with the research by Han et al. ${ }^{21}$ It is possible that nurses who have high levels of burnout cannot face life events calmly nor can they actively address the pressure and stressors. Our findings also suggested that a negative correlation exists between positive emotions and nurses' turnover intentions; in other words, when individuals have more positive emotions and use resources to face the challenges and opportunities of life, their life satisfaction is enhanced and their willingness to quit is reduced. It is possible that positive emotions can enhance individuals' positive adaptations to frustration and their ability to cope effectively with challenges, give positive meaning to stressful events, enable working closely with others, and help actively solve core problems. ${ }^{22}$

The proposed mediating effects of positive emotion were also supported by our data. The results showed that positive emotion mediated the relationship between burnout and nurses' turnover intention. This also suggested that positive emotions played an important role in burnout and turnover intention. Over time, the positive emotions experienced by individuals can be superimposed and can help to build many personal resources, such as enhanced positive beliefs, life goals, and social support, in addition to alleviating symptoms of illness. On the other hand, personal resources and enhanced individual life satisfaction reduce symptoms such as anxiety, helping the individual attain a higher level of positive emotions and psychological state, thus reducing the occurrence of job burnout and turnover intention. ${ }^{23}$

\section{Conclusions}

Positive emotion mediates the relationship between burnout and nurses' turnover intention in China. It is believed that nursing administrators should pay more attention to the benefits of positive emotion for working motivation. It is meaningful to relieve burnout in the working process so that nurses are willing to stay in their current employment.

\section{Conflicts of interest}

All contributing authors declare no conflicts of interest.

\section{Acknowledgments}

The authors gratefully acknowledge the supervisors and all employees who participated in this study for their assistance, as well as the experts and members of our group for their help and advice.

\section{References}

1. Leineweber $C$, Chungkham HS, Lindqvist $R$, et al. Nurses' practice environment and satisfaction with schedule flexibility is related to intention to leave due to dissatisfaction: a multi-country, multilevel study. Int J Nurs Stud. 2016;58:47-58.

2. Kovner CT, Djukic M, Fatehi FK, et al. Estimating and preventing hospital internal turnover of newly 
licensed nurses: a panel survey. Int J Nurs Stud. 2016;60:251-262.

3. Chiang YM, Chang Y. Stress, depression, and intention to leave among nurses in different medical units: implications for healthcare management/ nursing practice. Health Policy. 2012;108:149-157.

4. O’Brien-Pallas L, Murphy GT, Shamian J, Li X, Hayes LJ. Impact and determinants of nurse turnover: a pan-Canadian study. J Nurs Manag. 2010;18:1073-1086.

5. Heinen MM, van Achterberg T, Schwendimann R, et al. Nurses' intention to leave their profession: a cross sectional observational study in 10 European countries. Int J Nurs Stud. 2013;50:174-184.

6. Ayalew F, Kols A, Kim YM, et al. Factors affecting turnover intention among nurses in Ethiopia. World Health Popul. 2015;16:62-74.

7. Han K, Trinkoff AM, Gurses AP. Work-related factors, job satisfaction and intent to leave the current job among United States nurses. J Clin Nurs. 2015;24:3224-3232.

8. Shirom A, Melamed S. A comparison of the construct validity of two burnout measures in two groups of professionals. Int J Stress Manag. 2006;13:176-200.

9. Hong $E$, Lee YS. The mediating effect of emotional intelligence between emotional labour, job stress, burnout and nurses' turnover intention. Int $J$ Nurs Pract. 2016;22:625-632.

10. Zheng CF, Zhu HL. An analysis on 515 nursing staff's turnover intention. Chin Nurs Manag. 2007;7:29-31. (in Chinese).

11. Reivich K, Gillham JE, Chaplin TM, Seligman MEP. From Helplessness to Optimism: The Role of Resilience in Treating and Preventing Depression in Youth: Handbook of Resilience in Children. 2nd ed. New York, US: Springer; 2013:201-214.

12. Tang MQ, Zou WS, Qu HY. Characteristics of job burnout among nurses and its relations with subjec- tive well-being. China J Health Psychol. 2016;24: 40-44. (in Chinese).

13. Cohn MA, Fredrickson BL, Brown SL, Mikels JA, Conway AM. Happiness unpacked: positive emotions increase life satisfaction by building resilience. Emotion. 2009;9:361-368.

14. Wen YM, Gao ZL, Fang WT. The relationship between nurses's psychological capital and turnover intention as well as that nurse's burnout. China Health Standard Manag. 2017;8:6-9. (in Chinese).

15. Li CP, Shi K. Effects of distributional justice and procedural fairness on job burnout. Acta Psychol Sinica. 2003;35:677-684. (in Chinese).

16. Li X, Guan L, Chang H, Zhang B. Core self-evaluation and burnout among Nurses: the mediating role of coping styles. PLoS One. 2014;9:e115799.

17. Luthans KW, Jensen SM. The linkage between psychological capital and commitment to organizational mission: a study of nurses. J Nurs Adm. 2005;35:304-310.

18. Qiu L, Zheng X, Wang YF. Revision of Positive Emotional Negative Feelings Scale (PANAS). Chin J Appl Psychol. 2008;14:249-254. (in Chinese).

19. Wen ZL, Zhang L, Hou JT, Liu HY. The mediation effect of inspection procedures and its application. Acta Psych Sinica. 2004;36:614-620. (in Chinese).

20. Preacher KJ, Leonardelli GJ. Calculation for the Sobel test: an interactive calculation tool for mediation tests. http://www.quantpsy.org/sobel/sobel.htm. 2001.

21. Han S, Kim O, Joo Y, Choi E, Han J. Effects of nurses' mentoring on turnover intention: focused on the mediating effects role stress and burnout. $J$ Korean Acad Nurs. 2013;43:605-612. (in Korean).

22. Orehek E, Bessarabova E, Chen X, Kruglanski AW. Positive affect as informational feedback in goal pursuit. Motiv Emot. 2011;35:44-51.

23. Jin TT, Zhang LG. The impact of positive/negative emotion and humor styles on nurses' job satisfaction. Chin J Nurs. 2015;50:450-454. (in Chinese). 